\title{
Transformation With a JrVTE1 Gene for Tocopherol Cyclase Increasing the Content Of Total Tocopherol in Sour Jujube (Zizyphus jujuba Mill.var. spinosus Hu.)
}

\author{
Guan Qiuzhu \\ Shandong Institute of Pomology \\ No. 66 Longtan Road, Tai'an, Shandong, China \\ Tel: 86-538-826-6372Ｅ-mail: guanqiuzhu@163.com
}

\author{
Sun Hongyan \\ Shandong Institute of Pomology \\ No. 66 Longtan Road, Tai'an, Shandong, China \\ Tel: 86-538-826-6372Ｅ-mail: 290198419@qq.com \\ Sun Qingrong (Corresponding author) \\ Shandong Institute of Pomology \\ No. 66 Longtan Road, Tai’an, Shandong, China \\ Tel: 86-538-826-6372Ｅ-mail: sdipss@163.com
}

Received: November 5, 2018 Accepted: December 20, 2018 Published: December 23, 2018

Doi: 10.5296/jab.v7i1.14101 URL: https://doi.org/10.5296/jab.v7i1.14101

\begin{abstract}
Tocopherol cyclase (VTE1) plays a key role in promoting the production of tocopherol and increasing vitamin E content in plants. JrVTE1 gene isolated and cloned from walnut was transformed into genome of sour jujube (Zizyphus jujuba Mill. var. spinosus Hu.) by Agrobacterium tumefaciens. Putative transgenic lines were checked by polymerase chain reaction (PCR). The content of tocopherol of the transgenic plants were determined by high
\end{abstract}


performance liquid chromatography (HPLC). Compared to the non-transgenic sour jujube plants, the total content of tocopherol in transgenic plants was markedly increased in all tested tissues including the stems and leaves.

Keywords: Sour jujube, Genetic transformation, $\cdot$ JrVTE1· Tocopherol

\section{Introduction}

Sour jujube (Zizyphus jujuba Mill. var. spinosus $\mathrm{Hu}$.) is a wild fruit tree originated from China, it is widely distributed in central and north of China (Li et al., 2000). Being rich in vitamin $\mathrm{C}$ and minerals in fruit and seed, sour jujube fruits can be used as edible food and traditional Chinese medicine. Sour jujube is good rootstock of cultivated jujube (Zizphus jujube Mill.), because it has high resistant to cold and drought, tolerant to saline-alkali and more developed roots.

There were a few reports on Agrobacterium-mediated transformation in jujube. Gu et al. (2008) reported the Agrobacterium-mediated transformation in winter jujube using shoot tips as receptors and the transformation frequency was 5.2\%; Luo et al. (2015) and Guo et al. (2016) reported the Agrobacterium-mediated transformation in 'Huping' jujube using stem segments as receptors and the genetic transformation rate was $1.52 \%$ and $1.83 \%$, respectively; He et al. (2003) reported that the transformation frequency of 'Chalingsha' jujube were $2.4 \%$ and $4 \%$ respectively when using young stem segments and hypocotyls as receptors. But Agrobacterium-mediated transformation of sour jujube has not been reported.

Vitamin E, synthesized only by photosynthetic organisms, is obtained mainly from vegetable oils, nuts, cereals, green vegetables, fruits and oil seeds, and $\gamma$-tocopherol is the major form in seeds. the tocopherol cyclase (TC, VTE1) has been reported to be the key enzyme that catalyzes conversion of 2,3-dimethyl6-phytyl-1,4-benzoquinone (DMPBQ) to $\gamma$-tocopherol and promotes the production of $\gamma$-tocopherol and the total vitamin E content (Kanwischer et al., 2005; Vidi et al., 2006; Wang et al., 2015). The purpose of this study is to develop genetically modified sour jujube plants that will improve the Vitamin E content of sour jujube fruit. For such genetic engineering, Tocopherol cyclase gene (JrVTE1) was isolated and cloned from walnut. A stable plant regeneration protocol from leaf explants of 'Taishan' sour jujube (Wang et al. 2009) had been established. Transgenic plants of JrVTE1 gene were obtained and HPLC analysis showed highly Vitamin E content expressed in stems and leaves.

\section{Materials and Methods}

\subsection{Plant material and Culture Condition}

In vitro shoot cultures of 'Taishan' sour jujube were proliferated and maintained on a multiplication medium (MM): MS basal medium containing $2 \mathrm{mg} / \mathrm{L} \mathrm{BA}, 0.4 \mathrm{mg} / \mathrm{L} \mathrm{IBA}, 30$ $\mathrm{g} / \mathrm{L}$ sucrose, the $\mathrm{pH}$ was adjusted to 5.8 before autoclaving $\left(120^{\circ} \mathrm{C}, 20 \mathrm{~min}\right)$. The cultures were grown at $25{ }^{\circ} \mathrm{C}$ with a photoperiod of 16-h light $\left(40 \mu \mathrm{mol} \mathrm{m} \mathrm{m}^{-2} \mathrm{~s}^{-1}\right.$, as provided by cool-white fluorescent light) and 8-h darkness.

2.2 Shoot Regeneration From Leaf Explants and Determination of Phytotoxic Levels of Selective Antibiotic 
Expanding young leaves were excised from 3- to 4-week-old proliferation shoot cultures, wounded by several transverse cuts, and placed on an induction medium I (IMI): WPM basal medium containing $1.0 \mathrm{mg} / \mathrm{L} \mathrm{TDZ}$ and $0.5 \mathrm{mg} / \mathrm{L}$ IBA. The leaf explants were cultured in dark condition for 4 weeks on IMI, and then transferred to induction medium II (IMII): WPM basal medium containing $0.5 \mathrm{mg} / \mathrm{L} \mathrm{GA} 3$ and $0.1 \mathrm{mg} / \mathrm{L}$ IBA and cultured under the $16 / 8 \mathrm{~h}$ photoperiod for shoot bud elongation.

In a separate study, expanding young leaves were cultured onto IMI containing different levels of Kanamycin monosulphate $(\mathrm{Km}): 5,10,25,50 \mathrm{mg} / \mathrm{L}$ for 4 weeks, then transferred onto IMII containing the same concentration of $\mathrm{Km}$ as IMI. $\mathrm{Km}$ was filter-sterilized and added to the autoclaved media after the media had been cooled to $50{ }^{\circ} \mathrm{C}$ prior to solidification. The appropriate level of $\mathrm{Km}$ for selection of transformants was determined as the lowest concentration that completely inhibited regeneration from non-transformed leaves or produced white shoot from micropropagation. The shoot regeneration rate was recorded after 8 weeks of culture.

\subsection{Agrobacterium-Mediated Genetic Transformation}

The target gene JrVTE1 was isolated from walnut (Juglans regia). JrVTE1 was cloned and recombined into vector pRI101 (TaKaRa, Dalian, China) to create vector pRI101-JrVTE1 and confirmed by sequencing, and neomycin phosphotransferase II (NPTII) was used as a marker gene for the selection of transgenic tissue (Figure 1). Agrobacterium strain AGL1 was used as the host for the vector pRI101-JrVTE1. The culture of A. tumefaciens was grown overnight $\left(200 \mathrm{rpm}, 28{ }^{\circ} \mathrm{C}\right)$ in liquid LB medium with $0.5 \%$ glucose. Bacterial cells were collected by centrifugation for $10 \mathrm{~min}$ at $1500 \mathrm{~g}$ and then pellet was re-suspended and diluted to OD600 value of 0.6 with liquid co-culture medium (liquid IMI containing $20 \mu \mathrm{M}$ of acetosyringone (AS).

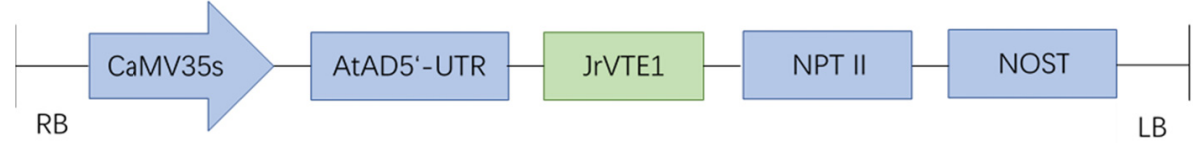

Figure 1. Schematic representation of T-DNA region of binary vectors pRI101

RB: right border; CaMV35S: cauliflower mosaic virus 35S promoter; AtAD50-UTR: the enhancer of JrVTE1 expression; JrVTE1: tocopherol cyclase gene; NPTII: neomycin phosphotransferase II; NOST: nopaline synthase gene terminator; LB: left border.

Expanding young leaves, wounded in advance, were immersed in bacterial suspension for 20-30 min with gentle shaking at $28{ }^{\circ} \mathrm{C}$. The infected leaves were blot dried on sterile filter paper to remove excess bacteria. Co-cultivation of Agrobacterium cell and leaf explants on liquid co-culture medium was carried out according to the method described by Sun et al. (2011) in the dark at $25^{\circ} \mathrm{C}$ for three days. After co-cultivation, the explants were washed 4-5 times with sterile deionized water and two times with liquid pre-selection medium (IMI with $100 \mathrm{mg} / \mathrm{L}$ carbenicillin (Carb) and $100 \mathrm{mg} / \mathrm{L}$ cefotaxime sodium salt (Cef), and then were 
blot dried and transferred to solid pre-selection medium for a three-day culture in the dark at $25{ }^{\circ} \mathrm{C}$. The explants were, in turn, incubated every one week onto selection medium I (pre-selection medium with $\mathrm{Km}$ ) till cultured 3 weeks before exposure to the light. The explants were then transferred to selective medium II (pre-selection medium with $\mathrm{Km}$ ) and cultured for 3 weeks before exposure to light, and then were transferred to selective medium II (IMII with $100 \mathrm{mg} / \mathrm{L}$ Carb, $100 \mathrm{mg} / \mathrm{L}$ Cef and $\mathrm{Km}$ ) and sub-cultured every 1 weeks till adventitious shoots formed. Theses adventitious shoots were transferred to MM with 100 $\mathrm{mg} / \mathrm{L} \mathrm{Carb}, 100 \mathrm{mg} / \mathrm{L} \mathrm{Cef}$ and $50 \mathrm{mg} / \mathrm{L} \mathrm{Km}$ for micropropagation and further selection to eliminate any remaining Agrobacterium cells. Every survived single shoot was individually marked and were propagated and gave rise to individual putatively transgenic lines. Transformation rate was calculated as the percentage of the Km-resistant green shoots (can grow and survive both on selection medium and MM containing $25 \mathrm{mg} / \mathrm{L} \mathrm{Km}$ ) that produced from total infected leaf explants.

\subsection{PCR Analysis of Transgenic Lines}

Genomic DNA was extracted from leaves of putative transgenic lines and non-transgenic (control) plantlets using a modified CTAB method. The sequences of the PCR primers were as follows:

\section{5'-CGCCATATGTCGAGGGGCCTATC-3'}

\section{5'-CGCGTCGACCTAAAGACCAGGGGGT-3'}

The PCR conditions were at $95{ }^{\circ} \mathrm{C}$ for $3 \mathrm{~min}, 30$ cycles at $94{ }^{\circ} \mathrm{C}$ for $20 \mathrm{~s}, 54{ }^{\circ} \mathrm{C}$ for $20 \mathrm{~s}$ and $72{ }^{\circ} \mathrm{C}$ for $2 \mathrm{~min}$, and the last step was at $72{ }^{\circ} \mathrm{C}$ for $5 \mathrm{~min}$. The amplification products were separated by electrophoresis on $1 \%$ agarose gels. Molecular weights were estimated using DNA marker DL2000 as a standard.

\subsection{Determination of Tocopherols by HPLC}

leaf and stem from transgenic plantlets were analyzed for tocopherols levels by HPLC performed on a LiChrospher Si-60 column $(5 \mathrm{~m}$ ) (Merck, Germany), using a UV detector (Shimadzu, 10AVP, Japan). Tocopherol ( $\alpha-, \beta-, \gamma$-and $-\delta$ ) was used as internal standard. Peaks of $\alpha$ - and $\gamma$ - in experimental samples were identified by comparison with elution times of standards. $\beta$ - and $\delta$-tocopherol was not detected in any tissue in this test.

\section{Results}

\subsection{Regeneration Potential of Leaf Segments Under the Stress of Km}

Sour jujube leaves were found to be very sensitive to the presence of $\mathrm{Km}$ in the medium. At lower $\mathrm{Km}$ concentration $5 \mathrm{mg} / \mathrm{L}$, the callus formation rate was reduced by $33.3 \%$, and shoot regeneration rate reduced by $80 \%$ (Table 1 ). $\mathrm{Km}$ concentration at $15 \mathrm{mg} / \mathrm{L}$, the leaf explants could form a small amount of callus blocks (callus formation rate was 7.14\%), but could not differentiate into adventitious buds. When the concentration of $\mathrm{Km}$ was higher than $25 \mathrm{mg} / \mathrm{L}$, the formation of callus and adventitious buds was completely inhibited, and the explant gradually became bleached and died. Therefore, $25 \mathrm{mg} / \mathrm{L} \mathrm{Km}$ was used for selecting putative 
transformed shoots during adventitious shoot regeneration from leaf explants.

Table 1. Sensitivity of leaf explants of sour jujube to kanamycin

\begin{tabular}{lll}
\hline Concentration of kanamycin $(\mathrm{mg} / \mathrm{L})$ & Callus induction rate $(\%)$ & Shoot induction rate $(\%)$ \\
\hline 0 & 100 & $96.7 \mathrm{c}$ \\
5 & 66.7 & $16.7 \mathrm{~b}$ \\
15 & 13.3 & $0 \mathrm{a}$ \\
25 & 0 & $0 \mathrm{a}$ \\
50 & 0 & $0 \mathrm{a}$ \\
\hline
\end{tabular}

\subsection{Genetic transformation and PCR Analysis of Putative Transgenic Plants}

Leaf tissues were inoculated with Agrobacterium, following co-cultivation, pre-selection, finally transferred to selection medium (Figure 2, A). Approximately $32 \%$ of the leaf explants produced excisable shoots $(2-3 \mathrm{~mm}$ ) on selection medium I (Figure 2, B). And then 23 regenerated Km-resistant shoots were obtained from the selection medium II (Figure 2, C). Theses shoots were transferred to MM with $\mathrm{Km}$ for micropropagation and further selection. It was found that a few shoots became white and could not survive under the stress of $\mathrm{Km}$ (Figure 2, D), finally died and discarded. Only $9 \mathrm{Km}$-resistant green shoots were obtained through the third selection.

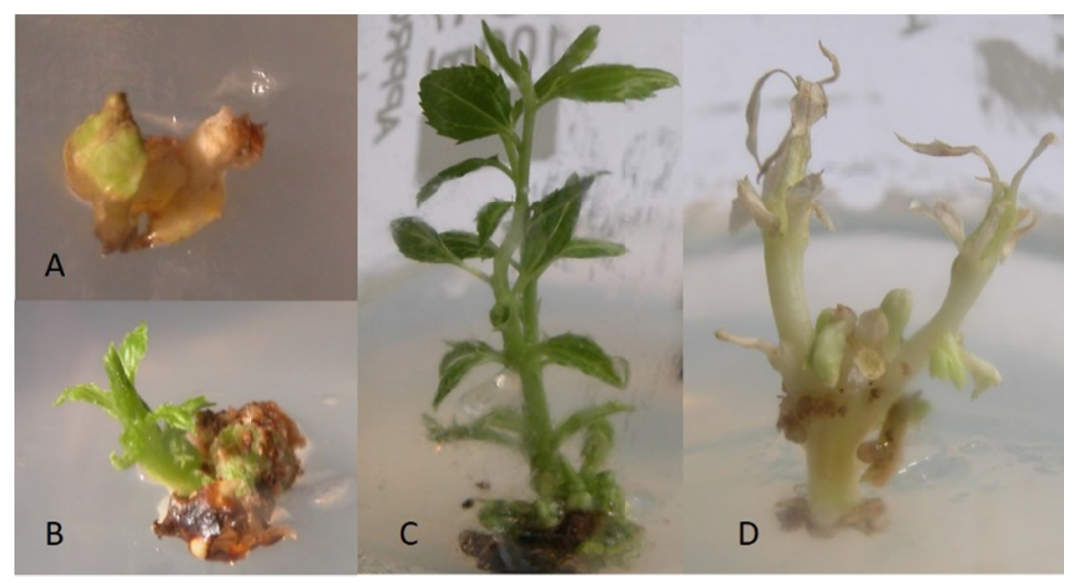

Figure 2. Plant regeneration from leaf explants of 'Taishan' jujube after infection with Agrobacterium

A. Leaf explant on selection medium I after co-cultivation. B. Shoot formation from the leaf explant on selection medium I after co-cultivation. C. Selected putative transformed plantlets. D. non-transgenic sour jujube shoot became white and died on MM containing Km. 
PCR analysis showed that a DNA fragment corresponding to the JrVTE1 gene (1353 bp) was amplified from genomic DNA isolated from leaves of some putative transformed plants (Figure 3, lanes1-3, 5). No amplification product was detected from DNA samples of the non-transformed control (Figure 3, lane 6). Results of PCR analysis preliminary proved that the JrVTE1 gene was integrated into the plant genome. Seven of 9 Kan-resistant plants were detected to be positive and the transformation rate was $5.83 \%$.

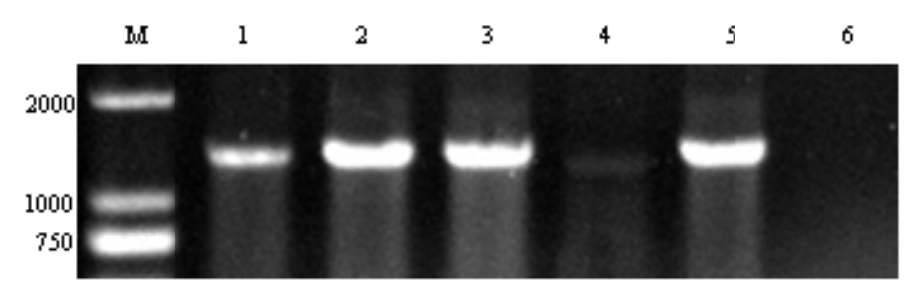

Figure 3. PCR analysis of putative sour jujube transgenic plants

Lane M: DL2000 DNA marker. Lane 1-5: putative transformed plants. Lane 6: non-transformed control.

\subsection{The Content of Tocopherol of Transgenic and Non-Transgenic Sour Jujube Plants}

Overexpression of JrVTE1 increased tocopherol content in tested tissues of the stems and leaves of transgenic sour jujube (Figure 4). The $\alpha$ - tocopherol content in the leaves and stem of transgenic lines were higher than non-transformed control. The $\alpha$ - tocopherol content was increased by $7.25 \mu \mathrm{g} / \mathrm{g}$ in leaf of transgenic sour jujube line 3 and by $3.04 \mu \mathrm{g} / \mathrm{g}$ in stem of transgenic sour jujube line 2, respectively. A small amount of or no $\gamma$-tocopherol was detected in stem of non-transformed control, but the $\gamma$-tocopherol content in stem of the 4 transgenic lines were range from 2.93 to $4.01 \mu \mathrm{g} / \mathrm{g}$. The total tocopherol content (sum of the content of $\alpha$ - and $\gamma$ - tocopherol) in the stem of the transformants was $30 \%-104 \%$ higher than the non-transformed control.

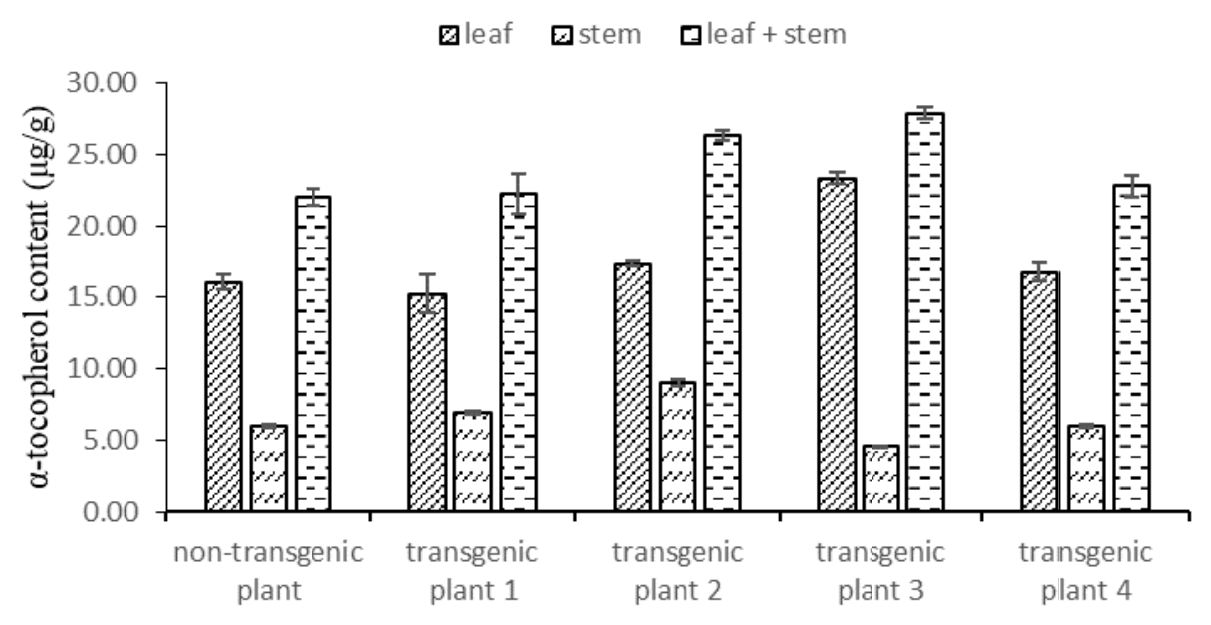



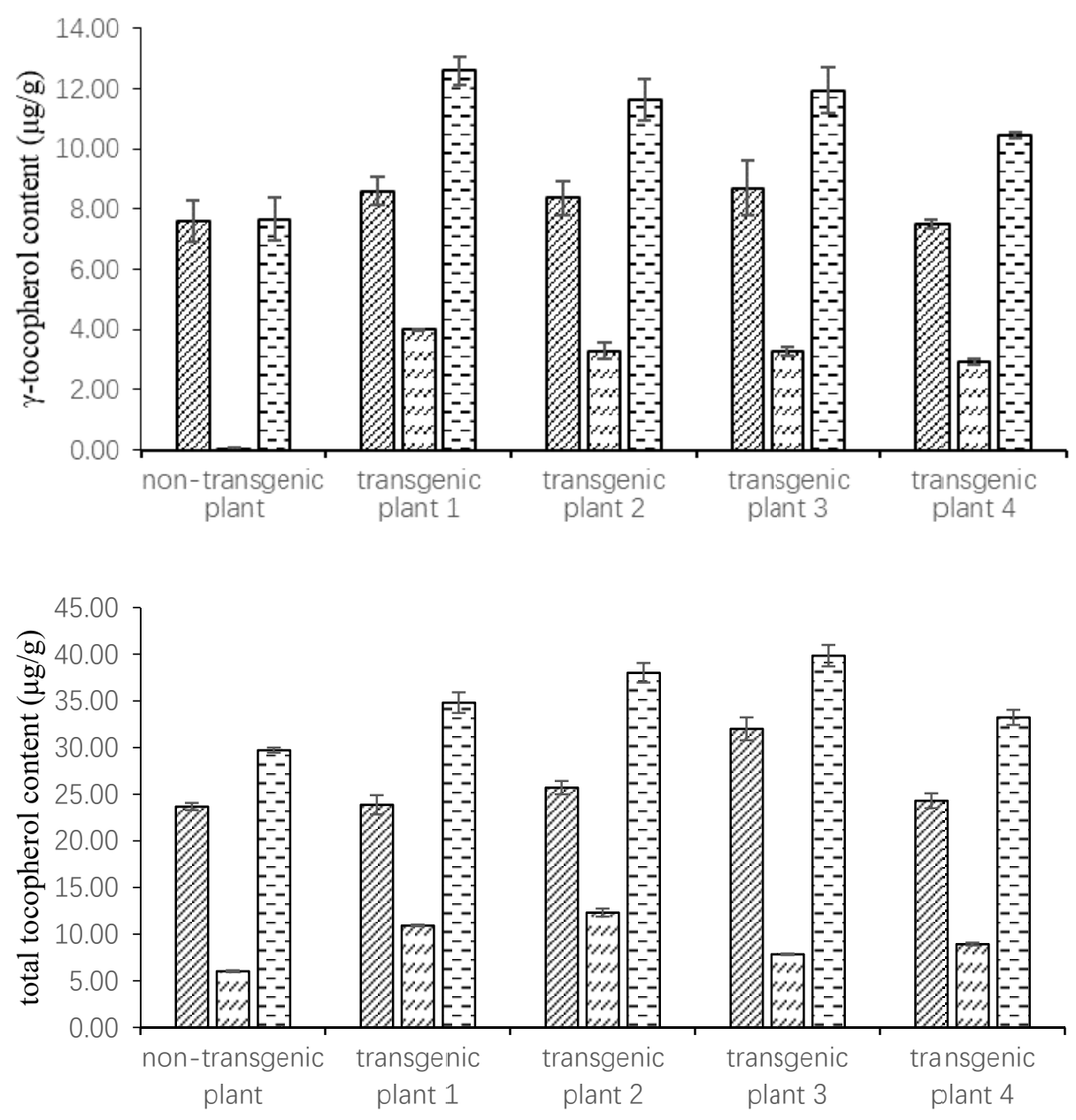

Figure 4. Tocopherol content of transgenic and non-transgenic sour jujube plants

\section{Discussion}

The present study established a protocol for Agrobacterium tumefaciens-mediated genetic transformation of sour jujube using leaf explants. Through the establishment of high frequency regeneration system of leaf and kanamycin sensitivity tests, a stable and efficient gene transformation receptor system for sour jujube was set up, which laid a sound foundation for Agrobacterium-mediated transformation by means of leaf disc method. In preliminary experiments, different basal medium, cytokine combinations and induction step were tested, and the high frequency shoot regeneration systems by two-step induction from leaf explants of 'Taishan' sour jujube has been established (Wang et al., 2009). The highest frequency of shoot regeneration was $100 \%$ by the two-step induction, and this shoot regeneration protocol was successfully used for Agrobacterium tumefaciens-mediated genetic transformation. Furthermore, in order to determine the appropriate concentration of selection agent to effectively screen transformed shoots, leaf explants were cultured on IMI and then on IMII supplemented with different concentrations of $\mathrm{Km}$. The formation of callus and adventitious buds was completely inhibited when the concentration of $\mathrm{Km}$ was higher than 25 $\mathrm{mg} / \mathrm{L}$, so the concentration of $\mathrm{Km}$ was maintained at $25 \mathrm{mg} / \mathrm{L}$ in all selection medium during 
the transformation experiments. Zhang et al. (2018) reported that $50 \mathrm{mg} / \mathbf{L ~ K m}$ was used for selecting putative transformed shoots generated from hypocotyls and cotyledons of 'Fengmiguan' jujube, while $125 \mathrm{mg} / \mathrm{L} \mathrm{Km}$ was used as the screening concentration of 'Jun' jujube callus (Feng et al., 2014).

For successful production of transgenic plantlets, co-cultivation is one of the most important steps for Agrobacterium-mediated transformation of plants. In the early study, we investigated the effect of co-cultivation medium state, liquid versus solid, on transformation efficiency of pear cultivar (Sun et al., 2011), and find out that co-cultivation with liquid medium yielded more green shoots and resulted in a higher transformation rate comparing to that obtained from solid co-cultivation medium. The same method of co-cultivation with liquid medium was also applied to the genetic transformation of sour jujube cultivar 'Taishan' and satisfied results are obtained.

In present study, $9 \mathrm{Km}$-resistant shoots were obtained through the selection and 7 of those plants were detected to be positive by PCR amplification, and the transformation percentage was $5.83 \%$. There were a few reports on Agrobacterium-mediated transformation in jujube, the transformation efficiency is not high enough to produce a large number of transgenic plants. He et al. (2003) reported that the regeneration rating of the adventitious buds from young stem segment and hypocotyls of 'Chalingsha' jujube were over 90\%, and the transformation frequency were $2.4 \%$ and $4 \%$ respectively. Gu et al. (2008) reported the Agrobacterium-mediated transformation of shoot tips of winter jujube and the transformation frequency was 5.2\%. Luo et al. (2015) and Guo et al. (2016) reported the Agrobacterium-mediated transformation of stem segment of 'Huping' jujube, and the genetic transformation rate was $1.52 \%$ and $1.83 \%$, respectively. In this study, the transformation with the JrVTE1 gene markedly increased the total content of tocopherol in the tested tissues including the stems and leaves of transgenic sour jujube. Previous studies reported that overexpressing VTE1 can enhance tolerance of plant to the drought (Liu 2008) and salt stress (Ouyang 2011). Therefore, abiotic stress tests of transgenic sour jujube plants overexpressing JrVTE1 gene would be carried out in our further work.

\section{Acknowledgement}

This work was supported by the Agricultural science and technology innovation project of Shandong Academy of Agricultural Sciences (CXGC2018E22).

\section{References}

Feng, X. D., Chen, G. L., Qiao, J., \& Chang, H. F. (2014). Study on Agrobacterium tumefaciens-mediated genetic transformation system for callus of Ziziphus jujube cv. Junzao. Journal of Yanan University (Natural Science Edition), 33, 54-57.

Gu, X., Meng, H. G., \& Zhang, J. (2008). Agrobacterium-mediated transformation of the winter jujube (Zizyphus jujuba Mill.). Plant Cell, Tissue and Organ Culture, 94, 23-32. https://doi.org/10.1007/s11240-008-9383-z

Guo, H. L. (2016). Construction, expression and catalytic activity identification of 4CL-STS 
fusion gene and its overexpression in ziziphus jujube Mill. [dissertation/ doctor's thesis]. [Beijing]: Beijing Forestry University.

He, Y. H., Lin, L. B., Xiong, X. H., He, G., Lin, S. Q., \& Chen J. H. (2003). Studies of development of efficient genetic transformation of Ziziphus jujube. Molecular Plant Breeding, $1,683-686$.

Kanwischer, M., Porfirova, S., Bergmuller, E., \& Dormann, P. (2005). Alterations in tocopherol cyclase activity in transgenic and mutant plants of Arabidopsis affect tocopherol content, tocopherol composition, and oxidative stress. Plant Physiol, 137, 713-723. https://doi.org/10.1104/pp.104.054908

Li, A., Zhang, G., Wang, X., Li, S., \& Shi, D. (2000). Preliminary report on biological characteristics and reproduction techniques of medical plant - Ziziphus jujube var. spinosa Hu. Inner Mongolia forestry Sci. and technol, 2, 29-32.

Liu, X. L., Hua, X. J., Guo, J., Qi, D. M., Wang, L. J., Liu, Z. P.,... Liu, G. S. (2008). Enhanced tolerance to drought stress in transgenic tobacco plants overexpressing VTE1 for increased tocopherol production from Arabidopsis thaliana. Biotechnol Lett, 30, 1275-1280. https://doi.org/10.1007/s10529-008-9672-y

Luo, Z., Guo, H., Yang, Y., Yang, M., Ma, L., \& Wang, Y. (2015). Heterologous overexpression of resveratrol synthase (PcPKS5) gene enhances antifungal and mite aversion by resveratrol accumulation. European Journal of Plant Pathology, 142, 547-556. https://doi.org/10.1007/s10658-015-0632-9

Ouyang, S. Q., He, S.J., Liu, P., Zhang, W. K., Zhang, J. S., \& Chen, S. Y. (2011). The role of tocopherol cyclase in salt stress tolerance of rice (Oryza sativa). Life Sci, 2, 181-188. https://doi.org/10.1007/s11427-011-4138-1

Sun, Q. R., Zhao, Y., Sun, H. Y., Hammond, R. W., Davis, R. E., \& Li, X. (2011). High-efficiency and stable genetic transformation of pear (Pyrus communis L.) leaf segments and regeneration of transgenic plants. Acta Physiol Plant, 33, 383-390. https://doi.org/10.1007/s11738-010-0557-z

Vidi, P. A., Kanwischer, M., Baginsky, S., Austin, J. R., Csucs, G., Dormann, P., Kessler, F., $\&$ Brehe lin, C. (2006). Tocopherol cyclase (VTE1) localization and vitamin e accumulation in chloroplast plastoglobuli lipoprotein particles. $J$ Biol Chem, 16, 11225-11234. https://doi.org/10.1074/jbc.M511939200

Wang, Y., Sun, Q. R., Wang, Y. Z., Li, X., \& Zhou. G., F. (2009). Establishment of regeneration system in vitro leaves of 'Taishan' sour jujube. Acta Botanica Boreali-Occidentalia Sinica, 29, 2118-2122.

Wang, C., Li, C., Charles, A. L., Sun Q. R., Guo, X. F., \& Yang, K. Q. (2015). Molecular cloning and heterologous expression analysis of JrVTE1 gene from walnut (Juglans regia). Mol Breeding, 35, 222. https://doi.org/10.1007/s11032-015-0414-2

Zhang, Y. Z., Zhang, M. Y., Li, J. D., Tan, B., Wang, T. F., Zheng, X. B., ... Feng, J. C. (2018). 


\section{Macrothink}

Optimization of genetic transformation system and transformation of Bt gene for epicotyl and cotyledon from seedlings of jujube 'Fengmiguan' (Ziziphus jujuba Mill). Journal of Henan Agricultural University, 52, 43-49.

\section{Glossary}

AAB Device: an equipment for sky.

KKD Device: an equipment for shipping.

\section{Appendix}

Appendix 1. Survey Question for Mobilephone Marketing

\section{Copyright Disclaimer}

Copyright reserved by the author(s).

This article is an open-access article distributed under the terms and conditions of the Creative Commons Attribution license (http://creativecommons.org/licenses/by/3.0/). 